\title{
Écriture et mnémotechnie : l'exemple des Cahiers Dux et Vénusté
}

\section{Guillaume Perrier}

\section{(2) OpenEdition}

\section{Journals}

Édition électronique

URL : http://journals.openedition.org/genesis/1145

DOI : 10.4000/genesis. 1145

ISSN : 2268-1590

\section{Éditeur :}

Presses universitaires de Paris Sorbonne (PUPS), Société internationale de génétique artistique littéraire et scientifique (SIGALES)

\section{Édition imprimée}

Date de publication : 15 juin 2013

Pagination : 65-77

ISBN : 978-2-84050-893-9

ISSN : $1167-5101$

\section{Référence électronique}

Guillaume Perrier, «Écriture et mnémotechnie : l'exemple des Cahiers Dux et Vénusté », Genesis [En ligne], 36 | 2013, mis en ligne le 08 juillet 2015, consulté le 19 avril 2019. URL : http:// journals.openedition.org/genesis/1145; DOI : 10.4000/genesis.1145 


\title{
Écriture et mnémotechnie : l'exemple des Cahiers Dux et Vénusté
}

\author{
Guillaume Perrier
}

$\mathrm{C}$ omment Proust a-t-il pu maîtriser la masse de ses manuscrits, dès lors qu'ils eurent atteint une certaine quantité ? Comment a-t-il pu se souvenir de tout ce qu'il avait écrit, de l'emplacement matériel où tel fragment avait été noté ou rédigé ? Émettons l'hypothèse que, face à une matière aussi foisonnante, la «mémoire naturelle » de l'écrivain n'ait pas suffi et qu'il ait eu recours - à partir d'un certain moment, par nécessité pratique - à la « mémoire artificielle1 », c'està-dire à divers procédés mnémotechniques. Cela suppose qu'il ait utilisé des procédés existants, ou qu'il ait inventé ses propres procédés. Pour envisager ces différents points, on peut prendre comme repère la date de 1913, année marquée non seulement par la publication de $D u$ côté de chez Swann mais aussi par l'invention de «l'épisode Albertine », qui inaugure un accroissement considérable de la matière romanesque ${ }^{2}$. On s'appuiera sur l'édition génétique des Cahiers 71 et 54 , dont la rédaction première remonte à 1913-19143. Parmi tous les procédés textuels ou graphiques que Proust a pu employer, on se limitera ici à deux types de signes linguistiques plus ou moins étudiés, mais dont la dimension mnémotechnique reste à établir : d'une part, les amorces de notes du type « capitalissime »; d'autre part, les noms de pages ou de cahiers.

Avant tout, qu'est-ce qu'un procédé mnémotechnique dans un manuscrit ? Comment définir, à l'intérieur d'une masse de documents écrits, certains éléments que l'écrivain adresserait spécifiquement à sa mémoire ? L'histoire des procédés mnémotechniques semble intrinsèquement liée à une tradition orale antérieure à l'invention de l'imprimerie, où la rareté des supports d'écriture impliquait de connaître les discours «par cœur » et de les prononcer de vive voix. De tels procédés, d'abord purement mentaux, s'adressaient moins aux écrivains qu'aux orateurs et aux récitants. Mais Frances Yates et ses successeurs ont montré que la tradition antique et médiévale de « l'art de la mémoire »s'est perpétuée après l'invention de l'imprimerie, dans une culture lettrée 4 . Ces systèmes de mémorisation, qui consistaient pour la plupart à inscrire des « images frappantes » dans des « lieux », n'ont jamais été aussi florissants qu'à la Renaissance. Ils se sont concrétisés dans les œuvres elles-mêmes, de l'Antiquité jusqu'à aujourd'hui. Les commentateurs ont identifié leurs traces aussi bien chez Joyce que Tite-Live, Dante ou Montaigne. Georges Perec a composé l'un de ses romans en s'appropriant un tel système. Dans une perspective génétique, la référence à

1. Distinction établie depuis l'Antiquité et toujours en vigueur dans la psychologie moderne et les sciences cognitives, même si l'artificiel se confond désormais avec la technologie informatique.

2. Voir Nathalie Mauriac Dyer, Proust inachevé. Le dossier « Albertine disparue », Paris, Champion, 2005, p. 34-37.

3. Marcel Proust, Cahier 54, éd. Francine Goujon, Nathalie Mauriac Dyer et Chizu Nakano, Paris, Bibliothèque nationale de France, Turnhout, Brepols Publishers, coll. «Marcel Proust. Cahiers 1 à 75 de la Bibliothèque nationale de France », 2008, 2 vol. ; Marcel Proust, Cahier 71, éd. Shuji Kurokawa, Pierre-Edmond Robert, Francine Goujon et Nathalie Mauriac Dyer, Paris, Bibliothèque Nationale de France, Turnhout, Brepols Publishers, coll. « Marcel Proust. Cahiers 1 à 75 de la Bibliothèque nationale de France », 2009, 2 vol.

4. Frances Yates, L'Art de la mémoire [1966], trad. Daniel Arasse, Paris, Gallimard, coll. «Bibliothèque des Histoires », 1975 ; Mary Carruthers, Le Livre de la mémoire. Une étude de la mémoire dans la culture médiévale [1990], trad. Diane Meur, Paris, Macula, coll. « Argô », 2002 ; Lina Bolzoni, La Chambre de la mémoire. Modèles littéraires et iconographiques à l'âge de l'imprimerie [1995], trad. Marie-France Merger, Genève, Droz, 2005. 


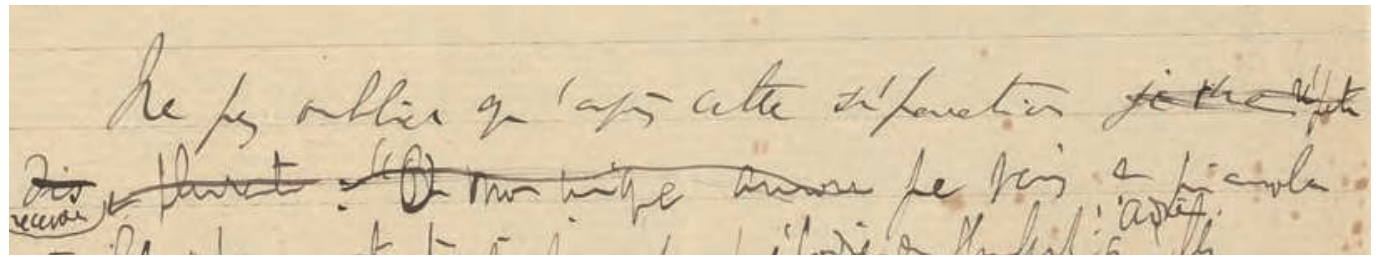

Fig. 1 : Cahier 71, fo 103 vo, détail (BnF, NAF 18321)

l'art de la mémoire a pu contribuer à éclairer certains manuscrits de Pascal et Stendhal ${ }^{5}$.

Plus fondamentalement, l'écriture peut être considérée en elle-même comme un procédé mnémotechnique. C'est la thèse de Socrate dans le Phèdre, présentée dans le mythe de l'invention de l'écriture par une divinité égyptienne, Theuth6. Tout signe écrit porterait la mémoire de ce qu'il signifie, souvenir répétable à volonté par la simple relecture. Socrate rejette cette mémoire extérieure, matérielle et prétendument inerte, au profit d'une mémoire intérieure et vivante, qui s'exprimerait par la parole. Mais on peut penser que certains signes écrits jouent un rôle particulier, qui redouble la fonction mnémotechnique de l'écriture. Ce sont des «suppléments 7 » de mémoire, nécessaires précisément lorsqu'il s'agit de mémoriser des documents écrits qui, en raison de leur masse, tendent à s'extérioriser et à se figer hors de la mémoire vivante, en un monument prématuré. Les procédés mnémotechniques de l'écrivain ne sont pas destinés à la conservation du passé mais doivent lui permettre de se réapproprier le déjà-écrit pour relancer le processus de création jusqu'à son terme, ou du moins vers son terme.

\section{Les amorces de notes}

L'expression d' «aide-mémoire », qui peut être employée dans le même sens que «procédé mnémotechnique », a le mérite d'attirer l'attention sur une pratique d'écriture spécifique. Elle se définit comme une « fonction opératoire attribuée à certaines notes “à ne pas oublier", souvent soulignées ou signalées graphiquement en marge, qui peut porter dans n'importe quel document de genèse sur des éléments dont l'écrivain diffère l'élaboration ${ }^{8}$ ». La note est effectivement le «supplément » privilégié de l'écrivain, celui qui tend à expliciter sa fonction mnémotechnique textuellement, lorsqu'il est précédé d'une amorce du type «ne pas oublier », et spatialement, lorsqu'il occupe la marge d'un manuscrit - ou les versos. Proust utilise généralement le recto pour le corps de sa rédaction; le verso est comme une marge supplémentaire, qui occupe presque plus de place que le corps du texte. La note se définit elle-même comme un "énoncé fragmentaire, souvent bref, qui peut être une simple notation (le résultat de l'acte de noter), et qui peut aussi se greffer sur un autre texte comme son commentaire et sa glose (une annotation) ${ }^{9} \gg$. En apparence, ces deux types de notes ne se ressemblent guère, mais avant cette double acception qui remonte au XVII ${ }^{\mathrm{e}}$ siècle, le terme « est lié depuis longtemps à la fonction mémorielle de l'écrit 10 », ainsi qu'à la tradition de l'art de la mémoire11.

Dans les Cahiers 71 et 54, Proust fait précéder certaines notes de la mention « ne pas oublier » qui vise explicitement sa propre mémoire. On en trouve une occurrence dans le Cahier 71, au folio 103 vo (voir fig. 1) : « Ne pas oublier

5. Catherine Baroin, Se souvenir à Rome. Formes, représentations et pratiques de la mémoire, Paris, Belin, 2010 ; Luigi De Poli, La Structure mnémonique de La Divine comédie, Frankfurt am Main, Bern et New York, Peter Lang, 1999 ; Daniel Martin, L'Architecture des Essais de Montaigne. Mémoire artificielle et mythologie, Paris, Nizet, 1992 ; Jacques Roubaud, «Hypothèses génétiques concernant la perecquation de la forme roman (communication du 6 juin 1993 au Séminaire Perec de Paris VII) », Le Cabinet d'amateur, no 4, 1995, p. 9-23 ; Daniel Ferrer, «Loci Memoriae: Joyce and the Art of Memory », dans Franca Ruggieri (dir.), Classic Joyce. Papers from the XVI International James Joyce Symposium, Rome 14-20 June 1998, Roma, Bulzoni editore, 1999, p. 355-360 ; Jean-Louis Lebrave, « Hypertextes - mémoires - écritures », Genesis, no 5, 1994, p. 9-24.

6. Platon, Phèdre [275 a], trad. Luc Brisson, suivi de Jacques Derrida, «La Pharmacie de Platon » [1968], Paris, Flammarion, coll. « GF », 2004, p. 178. Voir le commentaire de Jacques Derrida, ibid., p. 310-317.

7. Traduction envisagée par Jacques Derrida pour le grec pharmakon, rendu traditionnellement, selon les contextes, par «drogue » ou « remède » (ibid., p. 310-317); Platon l'emploie à propos de l'écriture. 8. Article «Aide-mémoire », préfiguration en ligne du Dictionnaire de critique génétique de l'ITEM, version du 21 décembre 2010 : <www.item. ens.fr/index.php?id=577448>.

9. Anne Herschberg Pierrot, « Les notes de Proust », Genesis, no 6, « Enjeux critiques », 1994, p. 61.

10. Ibid., p. 61.

11. Frances Yates, op. cit., p. 27, 55, 63, 330, 407 ; Mary Carruthers, op. cit., p. 162-163 ; Marc Fumaroli, L’Âge de l'éloquence. Rhétorique et "res literaria », de la Renaissance au seuil de l'époque classique, Genève, Droz, 1980, p. 259, n. 67.

12. Toutes les références à ces deux cahiers publiés chez Brepols peuvent se lire, pour chaque cahier, soit dans le volume I en fac-similé, soit dans le volume II en transcription diplomatique. Nous linéarisons ici cette dernière. 


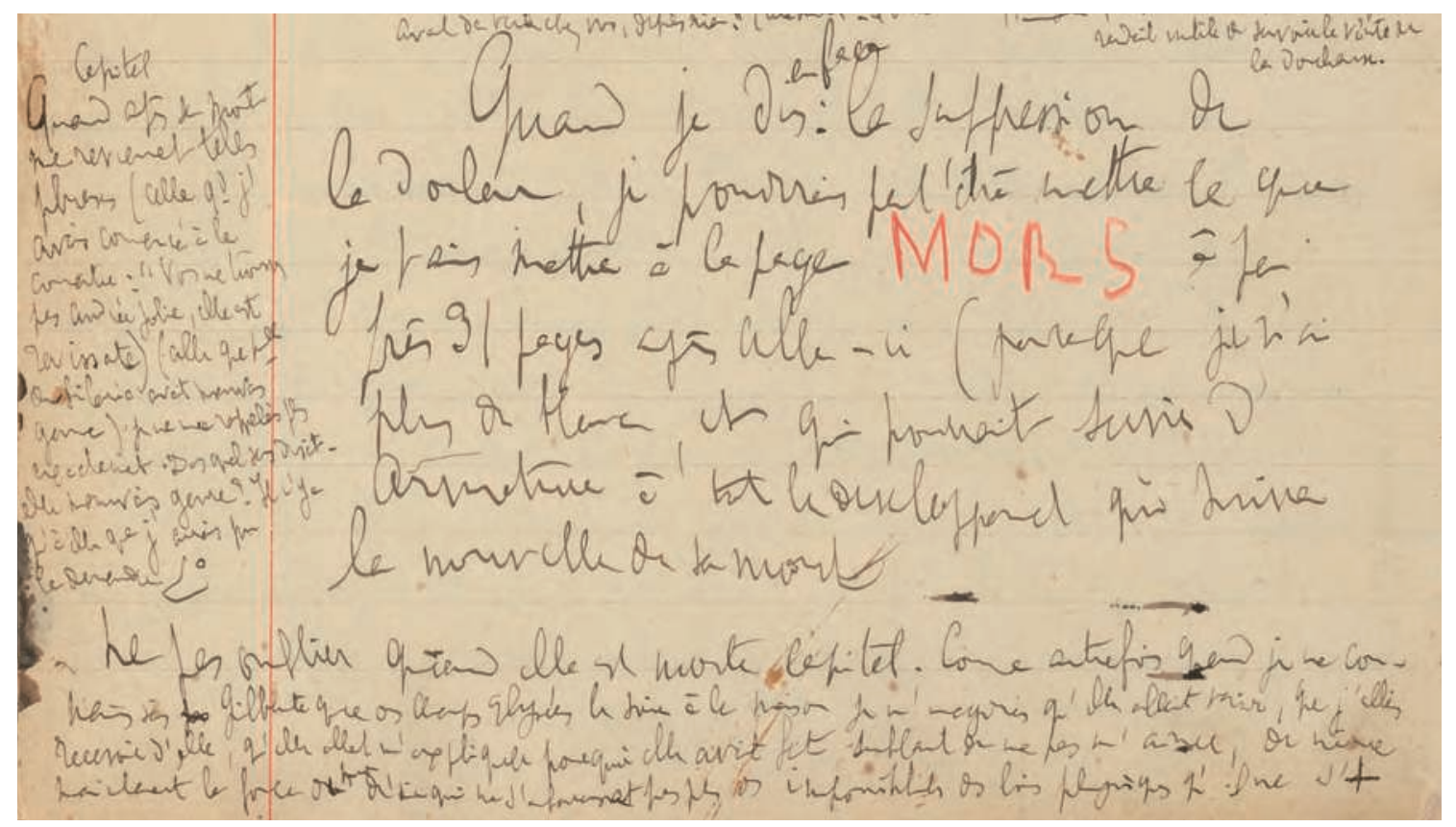

Fig. 2 : Cahier 54, fo 28 vo, détail (BnF, NAF 16694)

qu'après cette séparation je vais au pianola $[\ldots]^{12} »$. Deux apparaissent dans le Cahier 54, la première au folio $28 \mathrm{v}^{\mathrm{o}}$ (voir fig. 2) : « Ne pas oublier quand elle est morte Capital », amorce suivie d'une comparaison entre l'état d'esprit du héros-narrateur après la rupture avec Gilberte et après la mort d'Albertine ; la seconde dans la marge du folio 31 ro : «Ne pas oublier de montrer la solennité que prennent certaines de ses paroles et la phrase de sa lettre écrite sans doute sans y penser : je vous laisse le meilleur de moi-même. Et peut'être crépusculaire. »

L'expression « Ne pas oublier » pour introduire une note n'est pas la plus fréquente, loin de là. D'autres expressions qui jouent un rôle identique, quoique moins explicite, reviennent plus souvent : dix occurrences d'《 important » et « très important » dans le Cahier 71, neuf dans le Cahier 54, dont plusieurs sont soulignées ; six occurrences d'" essentiel » dans le Cahier 54, dont deux soulignées. Mais on sait que l'amorce favorite de Proust est l'adjectif «capital » ou « capitalissime ». On trouve seulement deux « capital » dans le Cahier 71, mais quarante-trois dans le Cahier 54, dont douze soulignés, ainsi que neuf « capitalissime », dont cinq soulignés. Il y a donc une démultiplication des emplois dans le Cahier 5413. On observe parallèlement une autonomisation de ces mots. Certaines occurrences, minoritaires, interviennent dans une structure syntaxique : «Il faudra marquer ceci qui est capital » (fig. 3), à savoir qu'Albertine est tantôt « immense », tantôt réduite à « presque rien » dans la pensée du héros

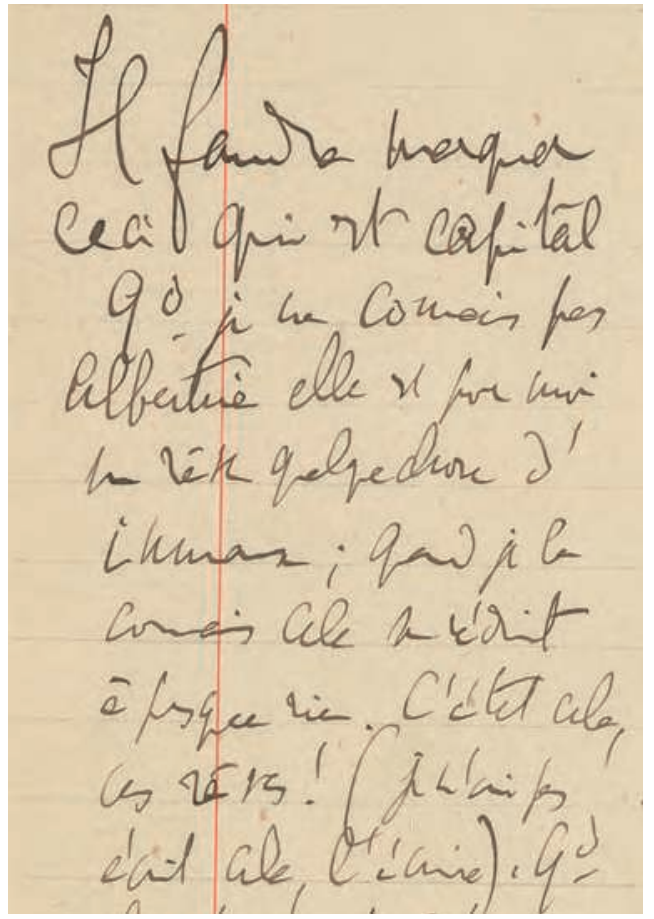

Fig. 3 : Cahier 71, fo 58 ro, détail (BnF, NAF 18321)

13. Florence Callu avance également la date de fin 1913 ; voir " "Capital, capitalissime", un mode de composition chez Marcel Proust ? », dans Almuth Grésillon et Michael Werner (dir.), Leçons d'écriture. Ce que disent les manuscrits. Hommage à Louis Hay, Paris, Minard, 1985, p. 81-82. 


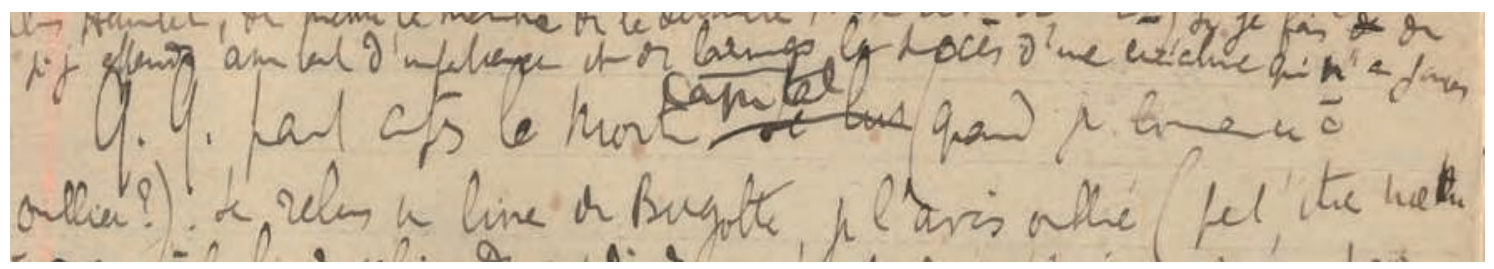

Fig. 4 : Cahier 54, fo 7 vo, détail (BnF, NAF 16694)

(Cahier 71, fo 58 ro, fig. 3). Les autres occurrences, très majoritaires, apparaissent hors de tout cadre syntaxique, soit comme celle que l'on a citée plus haut dans le même syntagme que « Ne pas oublier» (Cahier 54, fo 28 vo, fig. 2), soit, le plus fréquemment, isolées typographiquement sur la page, par exemple dans le Cahier 54, fo 7 vo (fig. 4) : « Capital », commençant «Q. Q. part après la mort », sur deux lignes différentes, en introduction d'une note sur les sentiments éprouvés par le héros pour des personnages de Bergotte, comparés à ses sentiments pour Albertine. Par leur caractère répétitif et asyntaxique, ces mots voient leur signification très affaiblie au profit de leur fonction purement signalétique ou, comme l'on essaye de le montrer, mnémotechnique.

Selon Florence Callu, « capital » et « capitalissime » jouent « un rôle d'aide-mémoire, de signaux à usage interne qui permettront à Proust, le moment venu, de retrouver ses notes ou de ne pas les retrouver [...] et d'en faire l'usage qu'il voudra14 ». On remarque en effet la liberté de l'écrivain dans ce domaine. Par exemple, au folio 9 vo du Cahier 71, en dessous de «Capital » isolé en tête, on peut lire une note qui commence ainsi : «En dehors des nombreuses Albertines simultanées (ou du moins remarquées pendant les mêmes vacances) il y aura les nombreuses Albertine qu'elle fut pour moi d'année en année. » L'idée est reprise dans plusieurs autres cahiers et notamment dans le Cahier 54, au folio $63 \mathrm{v}^{\mathrm{o}}$ et folio $86 \mathrm{vo}$, dans des additions beaucoup plus développées ${ }^{15}$. Mais l'abandon ou l'oubli n'est pas exclu : par exemple la note en marge des folios 51 ro et 52 ro du Cahier 54, relative au « doigt en crochet », geste obscène d'Albertine qui fait souffrir le héros quand il y repense après l'avoir oublié. Cet élément ne sera pas repris bien qu'il soit introduit ici par un «Capitalissime » souligné deux fois ${ }^{16}$. Dans les deux cas,
Proust note quelque chose qui doit être repris et développé dans une version ultérieure... si et seulement si l'occasion se présente. La mémoire artificielle semble rejoindre ici la mémoire involontaire.

Un autre aspect des manuscrits et de la formulation des notes est susceptible de confirmer cette idée. Anne Herschberg Pierrot esquisse une comparaison entre « l'espace-temps des cahiers » et l'art de la mémoire17. Elle remarque que la note de régie « attribue une place » au fragment qu'elle accompagne, mais aussi qu'il y a une «fragilité du fragment », un « caractère fondamentalement précaire des notations et de l'écriture qu'elles escortent ». La « durée de la genèse »n'est pas une mémoire totale, elle laisse place à un « temps de l'oubli18 ». En effet, certaines notes, même introduites par « capital » ou « capitalissime », sont accompagnées d'expressions aussi imprécises que « Mettre quelque part » ou « ailleurs ». Par exemple, dans une note que l'on a déjà citée à propos du détachement typographique du premier mot : «< $\underline{\text { Capital }}>/$ Q. Q. part après la mort ${ }^{19}$ » (Cahier 54, fo 7 vo, fig. 4). Ces amorces de notes ont donc, en définitive, un caractère non seulement répétitif mais imprécis. Elles ne visent pas à mémoriser définitivement,

\section{Ibid., p. 82.}

15. Voir les notes des éditeurs à ce sujet, Cahier 71, op. cit., p. 206.

16. Une autre occurrence de ce geste, au folio $57 \mathrm{ro}$, est biffée. Voir la note des éditeurs, Cahier 54, op. cit., p. 262.

17. Anne Herschberg Pierrot, "Proust et les notes de régie », dans Nathalie Mauriac Dyer, Kazuyoshi Yoshikawa (dir.), Proust aux brouillons, Turnhout, Brepols, coll. « Le champ proustien », 2011, p. 67 : «La Recherche, et ce n'est pas nouveau, s'écrit comme un art de la mémoire, des lieux et des figures de la fiction, mais aussi de l'espacetemps des cahiers. »

18. Ibid., p. 65 .

19. Cité par Anne Herschberg Pierrot, ibid., p. 64. 
à fixer prématurément quelque chose d'encore mobile et inabouti, mais à enregistrer matériellement et peut-être à oublier mentalement - si l'on peut dire - pour mieux se ressouvenir après coup.

\section{Les noms de pages et de cahiers}

On a pu se référer également aux théâtres de la mémoire de la Renaissance pour définir la mnémotechnie proustienne 20 . Ce modèle évoque l'espace physique et mental où l'écrivain crée son œuvre, où il peut embrasser du regard tous ses documents de travail, tout ce qu'il doit mémoriser. Le théâtre de Giulio Camillo ${ }^{21}$ ne donnait pas à voir directement le savoir universel qu'il prétendait contenir, mais des signes mnémotechniques qui permettaient de décomposer et de symboliser chaque composante de ce savoir. Dans le cas d'un écrivain comme Proust, les choses à mémoriser seraient les fragments discursifs plus ou moins longs qui constituent la matière de l'œuvre en devenir ; les signes seraient les noms inscrits, le cas échéant, sur la couverture de tel ou tel cahier, ainsi que les notes et les signes graphiques les plus visibles, qui permettent de localiser tel ou tel fragment, dans l'espace physique du cahier mais aussi dans l'espace virtuel de l'œuvre. À la différence des amorces de notes, les noms de pages ou de cahiers associent un mot singulier à une unité matérielle donnée. Ce sont des titres, ou plutôt des noms, qu'il faudrait replacer dans un ensemble très riche de « renvois génétiques 22 » d'une page à une autre, d'un cahier à un autre.

Dans le Cahier 54, on rencontre trois noms de page : la page «MORS », fo 60 ro (fig. 5), mentionnée au folio $28 \mathrm{v}^{\circ}$ (fig. 2), la page « Mac », fo 88 vo (fig. 6), mentionnée au folio 96 ro, et la page «Per», fo 102 ro (fig. 7), mentionnée plus haut, fo 69 ro - sans compter « la page Barimore du Cahier Vénusté » mentionnée dans le Cahier 56 (fo 24 vo), non localisée, sans doute détachée par Proust, puis perdue ou détruite. D'emblée, on remarque l'hétérogénéité de ces noms, mais aussi la brièveté et l'étrangeté apparente qu'ils ont en commun.

La page «MORS », qui en fait se prolonge sur cinq rectos, ne contient pas, comme on pourrait le croire, l'instant précis de l'annonce de la mort d'Albertine, mais une réflexion sur la persistance de la douleur après la mort de l'être aimé, une remise en cause de l'illusion de « la suppression de ma douleur » espérée à cette occasion. Elle contient également un rappel de la conversation avec Charlus qui renvoie à la première séquence du cahier (fos 1 vo à 9 ro) et dont toute l'importance apparaît rétrospectivement ici. Tout le passage introduit par ce nom «MORS »doit être intercalé «dans les pages qui commencent 31 pages plus haut et peut'être [ceci] commandera comme une armature tout ce qui suit la nouvelle de sa mort » (fo 60 ro). L'attribution d'un nom à cette page - ou ces pages - se justifie d'un point de vue pratique par l'importance de son contenu et par la nécessité d'opérer un remaniement important. En même temps que l'allusion érudite à un poème des Contemplations élucidée par les éditeurs 23 , notons le choix d'un mot latin et l'emploi des majuscules tracées au crayon de couleur rouge, de manière spectaculaire, non seulement sur la page ainsi nommée (fo 60 ro), mais aussi sur celle du renvoi (fo $28 \mathrm{v}^{\circ}$ ). L'emploi des couleurs, notamment du rouge, est un procédé traditionnel de l'ars memoriae et plus particulièrement de la composition des « images frappantes », procédé répertorié dans les traités antiques et médiévaux et employé par Giotto dans ses allégories de Padoue24. "MORS » pourrait donc être considéré comme une « image » destinée à rendre la page mémorable, en évoquant de manière fantasmatique et décalée la mort violente, peut-être le corps ensanglanté d'Albertine, en lien avec l'intensité de la douleur du héros.

20. Antoine Compagnon (dir.), Proust, la mémoire et la littérature, textes réunis par Jean-Baptiste Amadieu, Paris, Odile Jacob, coll. « Collège de France », 2009, p. 12-13 ; voir aussi Proust entre deux siècles, Paris, Éditions du Seuil, coll. «Poétique », 1989, p. 136.

21. Construction en bois ornée de nombreuses images (symboles religieux, figures mythologiques et astrologiques), aujourd'hui perdue, connue par la description et l'explication que son auteur en a données (Giulio Camillo, Le Théâtre de la mémoire [1550], trad. Eva Cantavenera et Bertrand Schefer, Paris, Allia, 2007).

22. Titre d'un index fourni par les éditeurs de chaque cahier Brepols (dans le volume II), outil indispensable aux analyses qui suivent.

23. Cahier 54, op. cit., p. 268.

24. Rhétorique à Hérennius, III, XXII ; cité par F. Yates, op. cit., p. 22. 


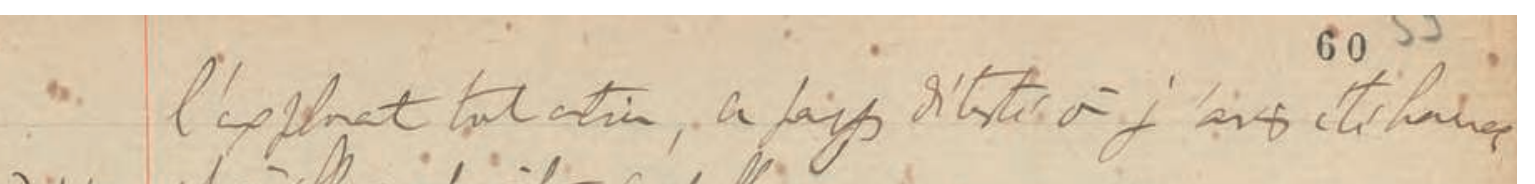

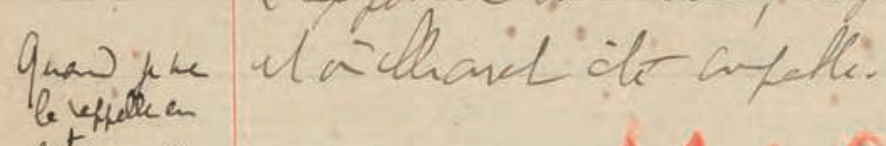

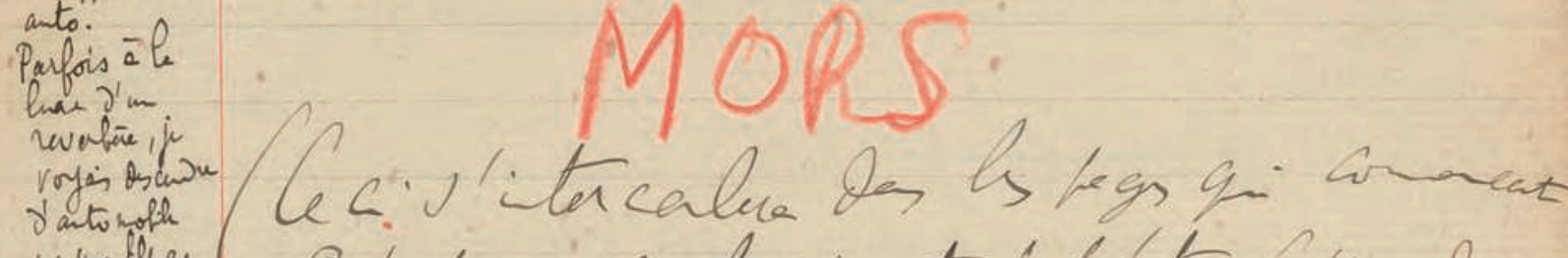

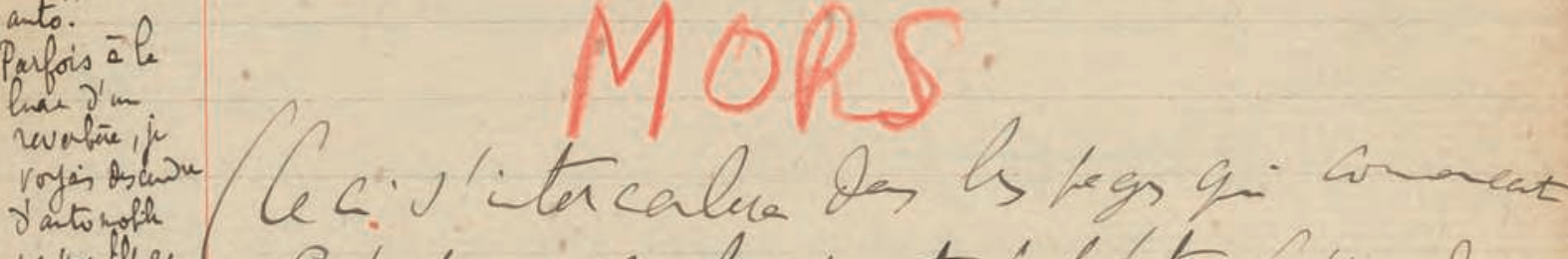

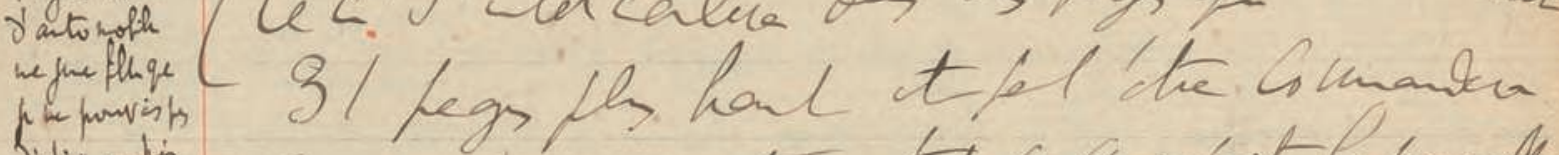

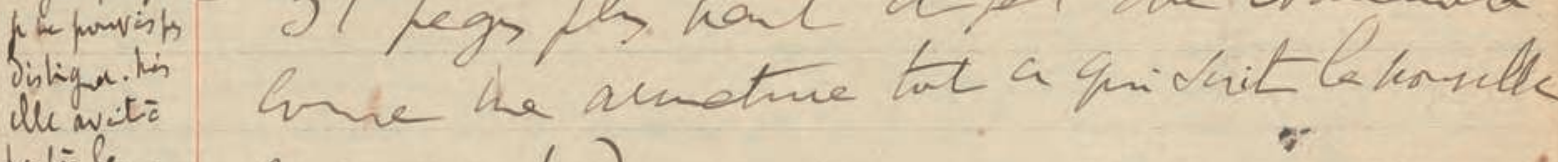

pingle. de ta hort)

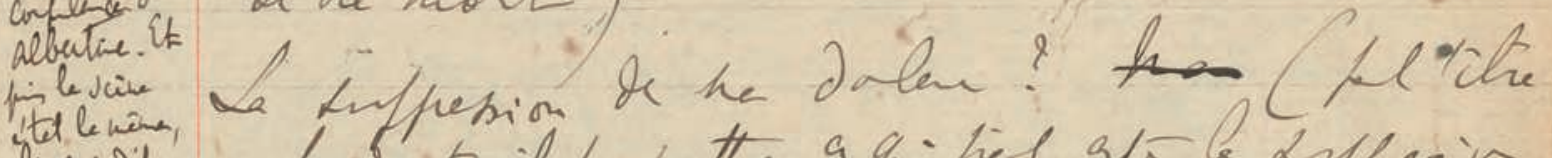

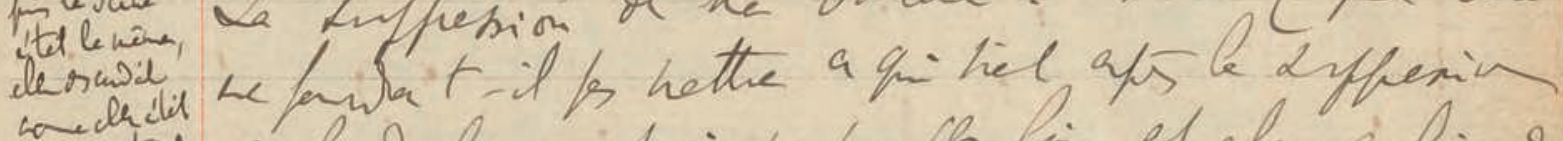

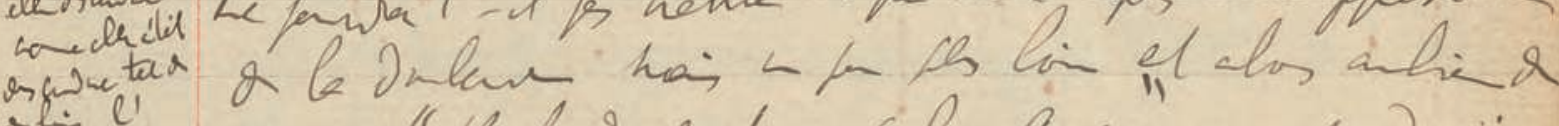

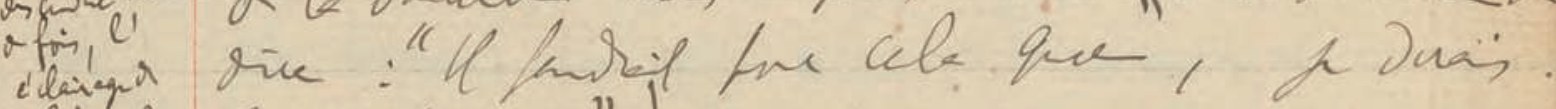

blebingark a Cratque ").

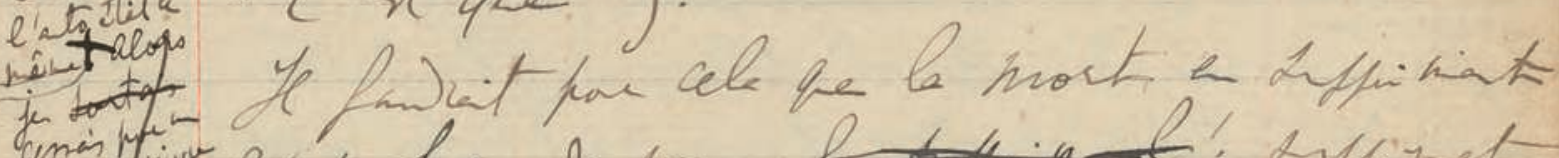

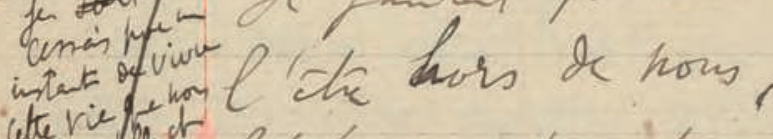

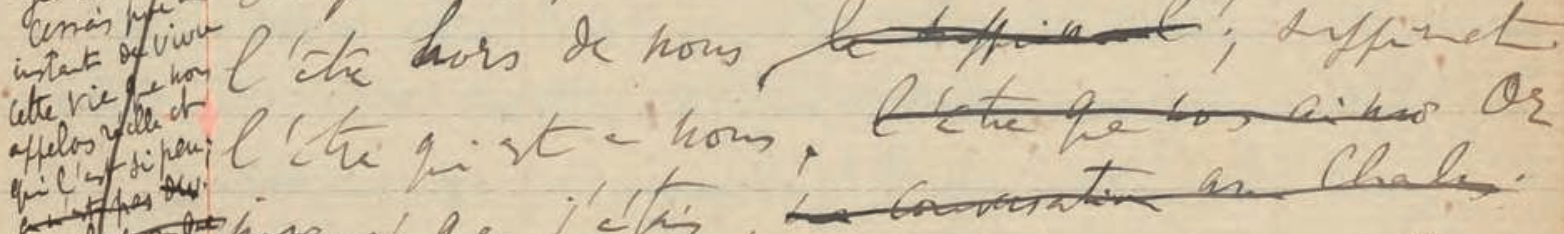

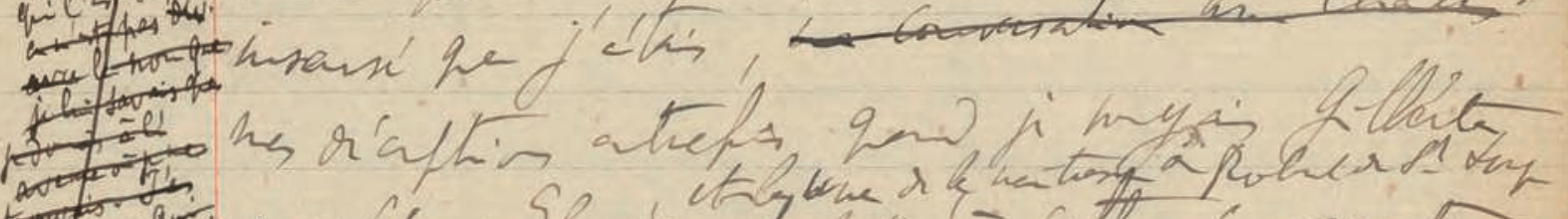

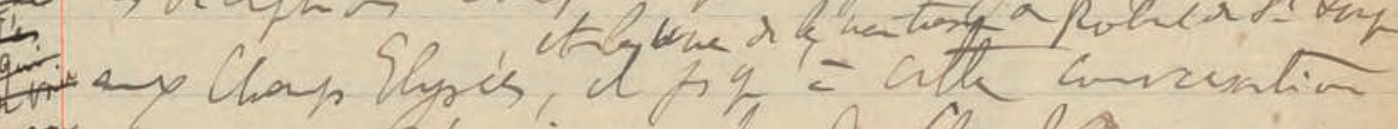

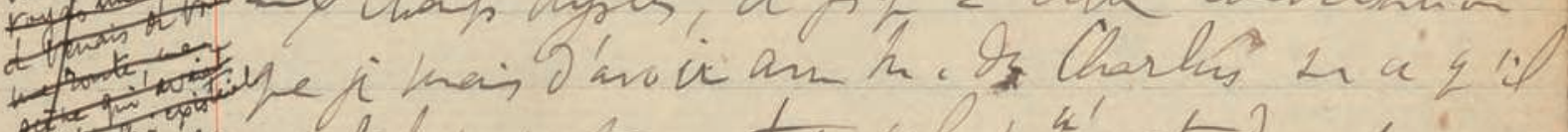

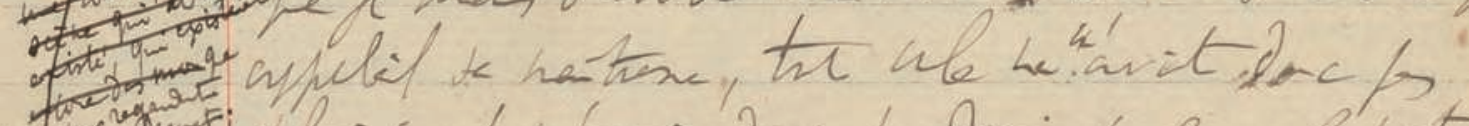

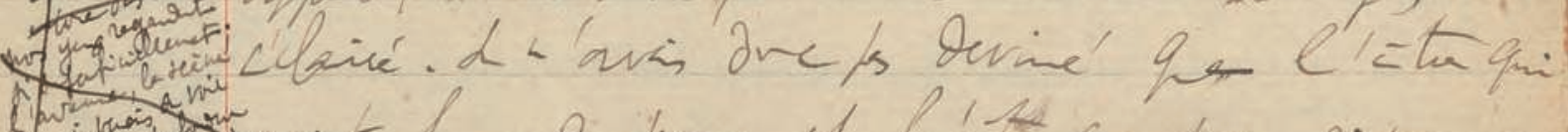

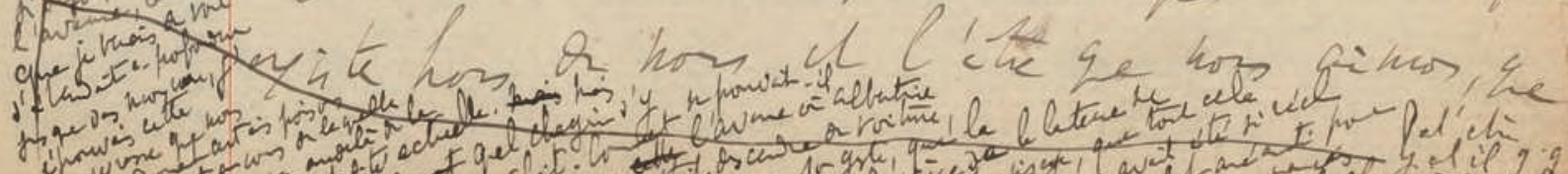

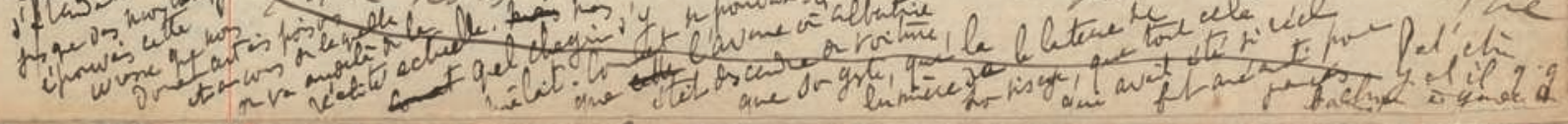

Fig. 5 : Cahier 54 , fo 60 ro
(BnF, NAF 16694$)$

(70) 


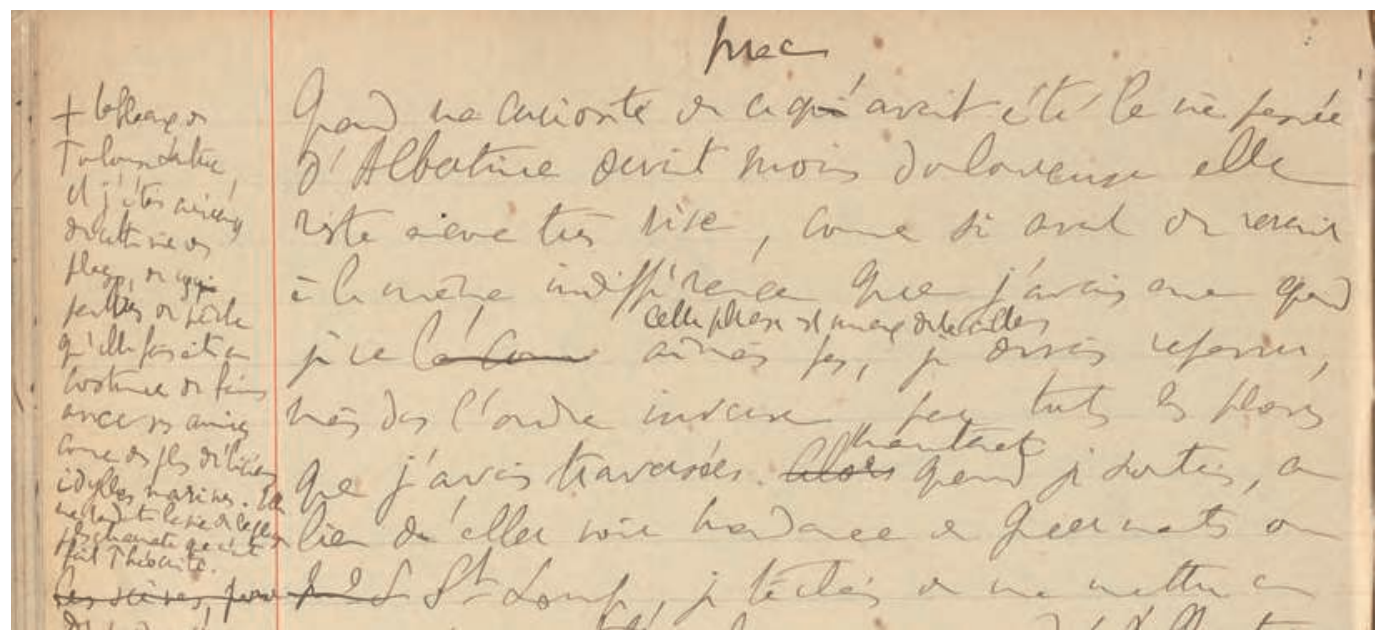

Fig. 6 : Cahier 54 , fo 88 vo, détail (BnF, NAF 16694)

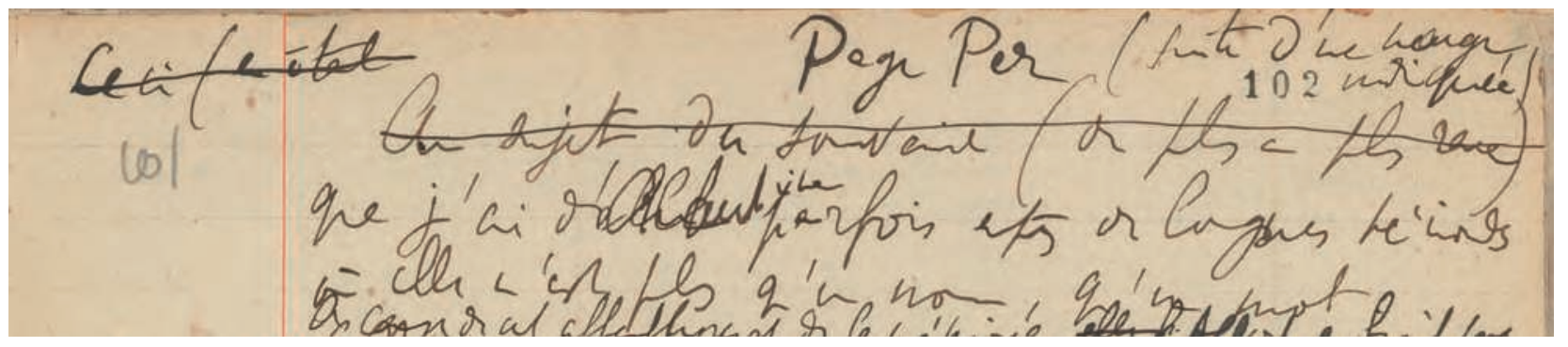

Fig. 7 : Cahier 54, fo 102 ro, détail (BnF, NAF 16694)

La page « Mac » et la page « Per », qui correspondent toutes les deux au récit du début de l'oubli, plus précisément à la recherche de nouvelles jeunes filles et à l'image d'Albertine enfermée dans un cachot souterrain, sont notées de manière plus sobre. Les noms sont simplement inscrits en tête de la page qu'ils désignent : «Mac» (fo 88 vo) et «Page Per (suite d'une marge indiquée) » (fo 102 ro). Ils sont intégrés dans une phrase lors du renvoi : «Peut'être ceci pourrait-il précéder la page Mac qui est au verso 7 pages avant » (fo 96 ro) et «Peut'être intercal[er] là la page Per qui est une des dernières du cahier » (fo 69 ro, en marge). L'initiale comporte une majuscule, comme il se doit pour un nom propre. Ces noms semblent choisis d'après l'amorce des pages en question, c'est-à-dire leurs premiers mots, voire dans un cas, d'après le dernier mot également. La page « Mac » commence ainsi : "Quand ma curiosité de ce qui avait été la vie passée d'Albertine devint moins douloureuse ». «Mac » est l'initiale du groupe sujet de cette proposition, isolée et extraite du syntagme propositionnel25. La page « Per », quant à elle, commence de la manière suivante, après une phrase qui permet de situer l'addition : «À cause de cet affaiblissement de la mémoire Albertine finit par devenir un simple mot, une personne pareille aux autres». « Personne » est le premier mot de la troisième ligne après la phrase introductive - sachant que les deux premières sont surchargées et difficiles à lire. La page se termine par « que mon imagination n'arrivait plus à percer»

25. Voir Julie André, « Le Cahier 46 de Marcel Proust : transcription et interprétation », thèse de doctorat, Université Paris III-Sorbonne nouvelle, 2009 , t. I, p. 29. 
(biffé), mots qui signifient l'impossibilité d'accéder aux profondeurs du moi où Albertine est enfermée. De « personne » et «percer », Proust tire les trois premières lettres, comme il l'a fait pour «ma curiosité ». Les noms obtenus sont monosyllabiques, comme « mors », mais ils sont encore plus courts et apparemment privés de sens. Ils évoquent simplement les mots dont ils sont l'initiale, ainsi que, pour «Per», la préposition latine qui n'est pas sans rapport avec le second de ces mots. Proust opère donc par prélèvement de lettres et par opacification du sens pour obtenir des noms opaques, brefs et percutants.

Mais les noms les plus frappants et les plus mystérieux sont ceux des cahiers eux-mêmes : «Dux » et «Vénusté », inscrits sur la couverture des Cahiers 71 et 54. Pour en éclaircir la signification, empruntons le détour de certains autres noms de cahiers, plus faciles à élucider. Proust donne lui-même la clef de l'appellation « cahier noir Serviette » pour désigner le Cahier 5726. Ce cahier qui contient une version précoce de la fin du roman, remontant à 1911 mais enrichie de notes 27 jusqu'en 1916 ou 1917, est désigné ainsi sur la couverture d'un cahier ultérieur. Cela tend à confirmer que les procédés mnémotechniques - si l'on veut bien considérer les noms de cahiers comme tels - ne sont pas apparus dès le début de la rédaction du roman. On lit sur la couverture du Cahier 74, lequel porte aussi un nom étrange que l'on ne tentera pas d'élucider ici mais qui a des connotations très riches, à la fois exotiques et familières : « Babouche / Et dans babouche plusieurs enclaves du dernier cahier noir Serviette qui finit le livre[,] enclaves que je n'ai pas eu la place de mettre dans le dit cahier noir (où il y a Serviette dans la 1re phrase) ». Le nom «Serviette » est donc simplement emprunté à la première page du cahier qu'il désigne. Comme dans les noms de pages, c'est l'amorce du contenu à mémoriser qui sert à désigner l'ensemble, à cette différence près que, ici, le mot ne semble pas choisi arbitrairement : il désigne le déclencheur d'une fameuse réminiscence du Temps retrouvé, et il doit lui-même déclencher le souvenir du contenu du cahier. La référence chromatique ne suffit pas toujours, car plusieurs cahiers ont la même couleur. C'est le cas des cahiers noirs à couverture moleskine dont Proust a utilisé un nombre assez important. D'où le recours à un élément supplémentaire. Récapitulons ici les règles de nomination du Cahier 57, qui sont au nombre de trois : couleur de la couverture, mot tiré du début du texte du cahier, mot désignant une réminiscence dans la fiction.

Un autre nom de cahier a été élucidé par les commentateurs. Il s'agit du Cahier 33, qui porte sur sa couverture la mention : «Cahier Fridolin ». En cherchant à dater la rédaction de ce cahier, Maurice Bardèche fait ce commentaire éclairant : «Cette dénomination patriotique indique une date vraisemblablement postérieure à octobre 1914 qui fut le moment où, par suite des détails donnés sur la "guerre de positions", l'uniforme feldgrau de l'infanterie allemande fut connu par le public, en même temps que le surnom "fritz" ou "fridolin" était donné aux soldats allemands 28 ». La couleur vert-gris des uniformes allemands ressemble en effet à celle des coins et du dos du cahier. Plusieurs remarques s'imposent ici : le glissement de la couleur du cahier à celle de l'uniforme et au surnom des soldats ; l'origine étrangère du nom, qui est déjà un nom propre ; l'allusion au contexte historique de guerre ; l'absence de rapports apparents entre le titre et le contenu du cahier, fragments relatifs à Albertine à Balbec. Pierre-Louis Rey conteste cette interprétation et en propose une autre29, en s'appuyant sur un passage du Cahier 51 (fo 68 vo), auquel il faut ajouter un passage du Cahier 57 (fo 40 ro) : le hérosnarrateur découvre l'apparence très vieillie du prince de Guermantes, alors qu'il se serait attendu à le voir en « costume du prince Fridolin 30 ». Il s'agit selon toute vraisemblance d'une référence à l' « opéra-bouffe-féerie » d'Offenbach et Victorien Sardou, Le Roi Carotte (1872), composé dans le

26. Publié dans Marcel Proust, Matinée chez la princesse de Guermantes. Cahiers du Temps retrouvé, éd. Henri Bonnet et Bernard Brun, Paris, Gallimard, 1982.

27. Notes riches en «Capital », « Capitalissime ». On trouve même un « [Ca]pitalissississimeissime» (ibid., p. 229) et un « capitalissime issime, issime » (ibid., p. 331).

28. Maurice Bardèche, Marcel Proust romancier, Paris, Les Sept couleurs, 1971, vol. II, p. 33. L'expression « dénomination patriotique » est bien sûr ironique. Mais à supposer que Proust ait éprouvé des sentiments germanophiles, c'est dans un contexte bien différent de celui où s'est tristement illustré Maurice Bardèche.

29. « Notice », RTP, II, p. 1327, n. 1. En ce qui concerne la datation, l'objection de Pierre-Louis Rey n'est pas nécessairement valide, car la nomination du cahier peut être postérieure à sa rédaction, si l'on considère que Proust nomme certains cahiers pour mieux les identifier, rétrospectivement.

30. RTP, IV, p. 874, 878 (« Esquisses »). 

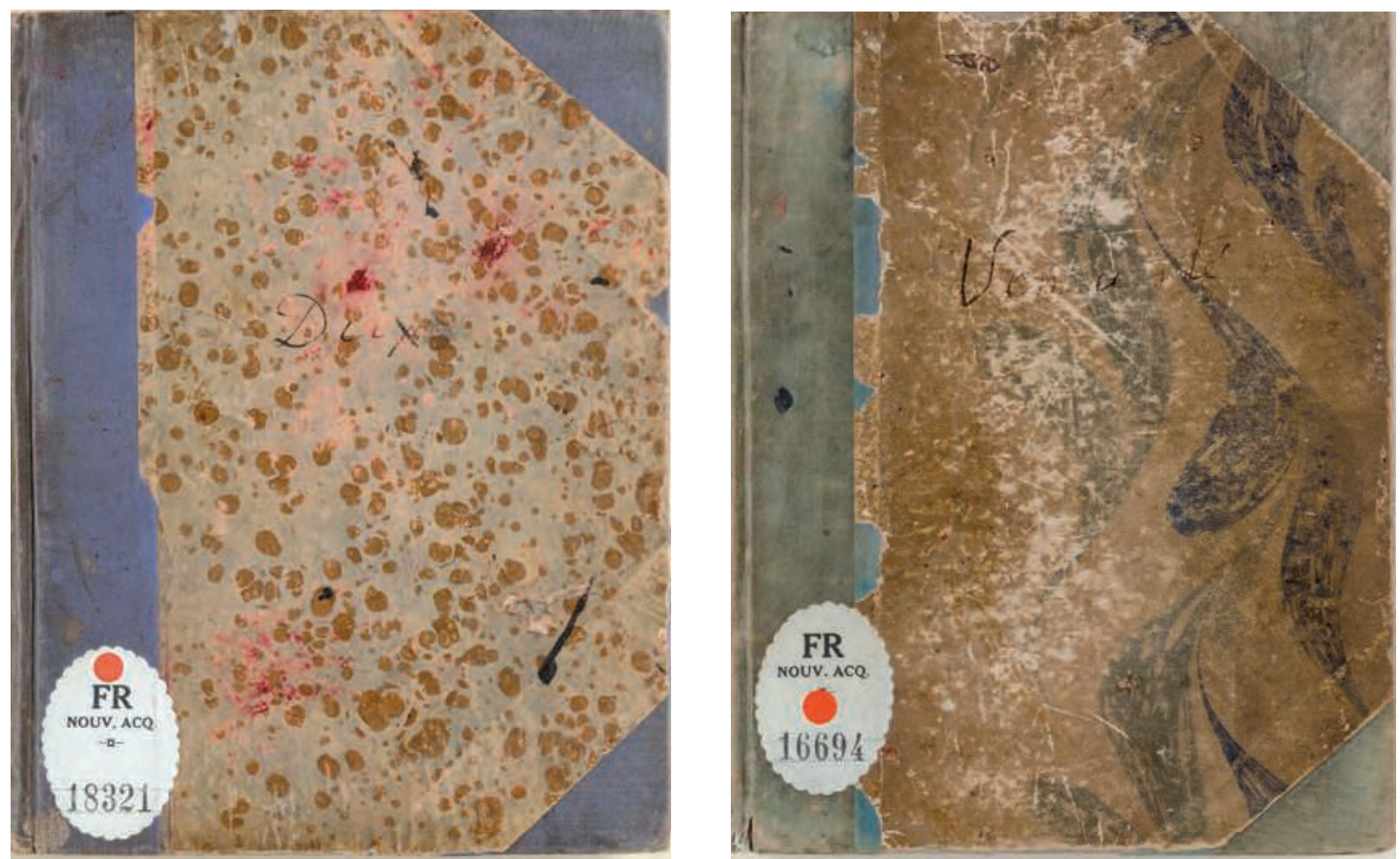

Fig. 8 : Plats de couverture des Cahiers Dux et Vénusté (BnF, NAF 18321 et 16694)

contexte de la guerre franco-prussienne. L'histoire est celle d'un jeune prince frivole, détrôné par un génie facétieux qui le remplace par un roi difforme, accompagné de ses courtisans à l'apparence de légumes. Les costumes dessinés par Théophile Thomas ont largement contribué au succès du spectacle 31 . La référence au personnage d'Offenbach n'est pas incompatible avec l'explication de Maurice Bardèche, surtout si l'on considère que, dans les deux cas, il est question de couleur de vêtements et de guerre francoallemande. Fridolin serait un nom susceptible de faire image dans la mémoire de l'écrivain, car surdéterminé et chargé de connotations se rapportant à la couleur du cahier. Les règles de formation du nom de ce cahier sont donc au nombre de deux : référence à la couleur de la couverture, allusion plaisante au contexte historico-culturel par l'intermédiaire d'un nom d'origine étrangère. Si l'on compare ces règles à celles du Cahier 57, on peut tenter de dégager les facteurs qui président aux choix de Proust. Le premier est assez stable et concerne l'aspect extérieur du cahier ou les premières lignes de son incipit. Le second est plus variable et renvoie à quelque chose de différent du cahier dans sa matérialité, par une allusion stimulante, soit sérieuse, soit plaisante. On peut penser qu'il en va de même pour les noms plus difficiles à élucider.

Les Cahiers 71 et 54 sont des cahiers «bibliophiles » (voir fig. 8), du même type que le «Cahier Fridolin ». Leur couverture est colorée, le plat étant de couleurs différentes de celle des coins et du dos. La présence de leur nom respectif, « Dux » et «Vénusté », non seulement

31. Voir Théophile Thomas, Maquettes de costumes pour le «Roi Carotte » (en particulier la planche 3 ), conservées à la Bibliothèque-musée de l'Opéra sous la cote D $216 \mathrm{Z}$ 1, et reproduites sur le site « Banque d'images » de la BnF. Les couleurs du plat du Cahier 33 ressemblent étrangement aux teintes variées du costume du prince Fridolin, mais il est très improbable que Proust ait eu accès, directement ou indirectement, à ces documents. Il n'y a aucune référence explicite au Roi Carotte dans les écrits de Proust mais il faut bien que l'écrivain ait entendu parler de ce spectacle pour qu'il emploie l'expression de « costume de prince Fridolin ». On trouve du reste certaines références ou allusions à Offenbach et Sardou, notamment dans la correspondance avec Reynaldo Hahn. 
sur la couverture mais dans des renvois génétiques d'un cahier à l'autre, sur des rectos 32 , laisse penser que, contrairement au Cahier 57 baptisé a posteriori, ces noms sont contemporains de la rédaction du corps principal du texte (1913-1914). On remarque que ce sont deux mots relativement opaques, empruntés - à deux degrés différents - à la langue latine. Les éditeurs formulent les hypothèses suivantes, concernant le premier des deux cahiers. D'une part, le sens latin de « Dux » pourrait faire référence au rôle de guide du Cahier 71, par rapport au nouveau scénario que Proust invente pour la suite de son roman. D'autre part, un texte de Ruskin mentionne l'inscription «DUX » sur une mosaïque de la basilique Saint-Marc. Dans cette mosaïque du transept nord, « le Doge est debout au milieu [des fidèles], reconnaissable à son bonnet cramoisi, brodé d'or et à l'inscription DUX, placé au-dessus de sa tête 33 ». Ces deux pistes interprétatives sont satisfaisantes. Elles entrent toutes deux dans la catégorie de la deuxième règle que l'on a définie. Pour envisager la première règle, on peut s'arrêter sur l'aspect matériel du cahier et tenter de le mettre en rapport avec l'une des deux interprétations. Or, l'aspect de la couverture de ce cahier est extrêmement frappant. Malheureusement, son état de dégradation actuel ne permet pas de dire dans quel état il était quand Proust l'a utilisé. Les coins et le dos du cahier du moins n'ont pas dû beaucoup changer de couleur, ils sont bleu-violet. Proust appelle effectivement ce cahier « Cahier violet Dux 34 ». C'est le plat de la couverture dont le chromatisme est le plus étrange : on observe des taches marron ou rousses, assez régulières, sur un fond beige-gris plus clair, deux teintes qui semblent appartenir à l'aspect premier du cahier ; on observe également des taches rouges, très irrégulières, concentrées plutôt dans la partie supérieure, et dans une moindre mesure dans la partie inférieure gauche. Le problème est de savoir si leur apparition est contemporaine de Proust. Si c'était le cas, le rapport entre la citation de Ruskin et l'apparence de la couverture serait renforcé, puisque, au plat tacheté de la couverture, qui peut rappeler la surface d'une mosaïque, s'ajouterait la présence d'une teinte rouge, qui rappellerait le « bonnet cramoisi du doge ». Les deux règles dégagées précédemment seraient alors observées : la règle constante matérielle (aspect de mosaïque, teinte rouge sur fond tacheté) et la règle variable (référence esthétique à Ruskin, au doge vénitien dans la mosaïque de Saint-Marc ${ }^{35}$, au rôle directeur d'un tel personnage et du scénario ébauché dans le cahier). L'ensemble constituerait à la fois un nom propre et une image frappante, dissemblable ${ }^{36}$.

Le nom le plus délicat à élucider est celui du Cahier 54, «Vénusté ». Les éditeurs donnent des éléments de définition de ce latinisme qui remonte au XVI siècle $^{37}$. Il s'agit d'intégrer ces données indispensables dans la démarche d'élucidation que l'on tente de mettre en place ici, en commençant par la première règle. «Vénusté » ne saurait référer à l'aspect de la couverture : coins bleuvert, plat marbré beige et vert. Il faut donc se reporter à l'incipit du cahier, comme pour le cahier « Serviette ». Rien dans les premiers mots n'évoque le terme « vénusté » mais, comme le signalent les éditeurs, on lit au folio 4 vo : «Dans le monde des sports car Félix avait pensé aussi à s'y consacrer quelque temps M. de Charlus avait cru que la Vénus masculine s'incarnait moins fréquemment ». «Vénusté » est donc susceptible d'évoquer toute la première séquence du cahier, des folios 1 à 9 , qui présente une forte unité et qui porte sur une conversation avec M. de Charlus et sur les amours malheureuses de ce dernier avec un certain Félix. Comme on l'a vu précédemment, le héros-narrateur s'apercevra plus tard de l'importance de cette conversation et des leçons qu'il aurait dû en tirer pour sa propre souffrance amoureuse - peu importe l'existence objective ou la nature de l'être aimé, c'est son image intérieure qui compte et qui fait souffrir. C'est donc un passage très important, plus important qu'il n'y paraît au premier abord, et c'est un passage qui place Vénus sous le signe de l'inversion sexuelle. Une seconde occurrence de Vénus dans le cahier confirme le thème de l'inversion sexuelle, concernant cette fois Albertine et Andrée

32. Cahier 71, fo 104 ro (en marge il est vrai); Cahier 54, fo 10 ro.

33. Voir John Ruskin, Les Pierres de Venise [1851-1853], trad. Mathilde Crémieux [1906], Paris, Hermann, coll. « Savoir », p. 54-55; cité par les éditeurs du Cahier 71 .

34. Carnet 3, fo 22 vo (voir «Index des renvois génétiques », Cahier 71, vol. II, p. 257).

35. Rappelons également que la basilique Saint-Marc est l'objet de la réminiscence des pavés inégaux dans Le Temps retrouvé.

36. Sur la dissemblance comme caractéristique des images frappantes, voir Georges Didi-Huberman, Fra Angelico. Dissemblance et figuration, Paris, Flammarion, 1990.

37. Voir Cahier 54, vol. II, p. 237. 
(fo $40 \mathrm{v}^{\mathrm{o}}$ ). On comprend donc l'importance de la figure mythologique et de ses retournements pour ce cahier, à la fois pour la séquence Charlus et pour la séquence Albertine. Mais pourquoi «Vénusté », si ce n'est l'abstraction et la préciosité produites par le suffixe ? Un détour par l'histoire de la langue et par les occurrences littéraires du mot n'est pas superflu ${ }^{38}$. Il s'en dégage l'impression d'un mot profondément ambigu, à la fois noble par son origine et vulgaire par la sorte de beauté sensuelle qu'il peut désigner. Cette noblesse sert le cas échéant à voiler ou à suggérer une forme de sensualité inavouable. Dans une lettre de 1907 à Reynaldo Hahn signalée par les éditeurs du Cahier 54, Proust écrit d'une sœur de Mme Greffulhe : «Sa petitesse et sa vénusté sont d'ailleurs très comiques et font penser à quelque beauté parfaite et minuscule comme on n'en voit l'étrangeté que dans certains bordels ${ }^{39} »$. Proust a pu relever ce mot dans la langue de son époque, dans une conversation ou chez un écrivain de son temps. Des occurrences plutôt conventionnelles, au sens de beauté gracieuse, sont répertoriées chez Ernest Renan, Maurice Barrès et Anatole France ${ }^{40}$. Mais l'emploi le plus intéressant, dans un livre dont on sait que Proust l'a lu et qu'il en a reçu une forte impression, fût-elle désagréable, se trouve dans le recueil pornographique de Verlaine, Femmes (1890), dédié aux « Putains », « novices ou professes ». On lit dans le premier poème, « À celle que l'on dit froide » : «[...] ta gorge triomphante / Dans sa gracile vénusté ». Deux strophes plus loin, la jeune femme est comparée à « un joli garçon ». Nombreuses sont les comparaisons entre filles et garçons dans ce recueil qui constitue un diptyque avec Hombres (1891), consacré aux amours masculines. Proust aurait acquis ces deux livres lors d'une vente de 1908, d'après Philip Kolb, et il en parle dans deux lettres de la même année, en les qualifiant de «scandaleux », « immondes », « secrets », « stupides », et encore quatorze ans plus tard, en 1922, en employant les termes « hideux » et «pénible 41 ». Si cette inspiration verlainienne est avérée, alors on comprend mieux la charge émotionnelle et le caractère transgressif de "vénusté », susceptibles d'en faire une image frappante et un titre adéquat pour le Cahier 54. Ce mot condenserait la passion amoureuse de Charlus pour Félix et celle du héros pour Albertine, et refléterait les tourments de Proust lui-même. Il véhiculerait une puissance comparable à celle que Bataille a conférée au mot « érotisme » et correspondrait bien au procédé indiqué par l'auteur de la Rhétorique à Hérennius : « créer des images capables de rester le plus longtemps possible dans la mémoire », « des images qui ne soient ni nombreuses ni vagues mais actives », qui doivent avoir « une beauté exceptionnelle ou une laideur particulière $42 »$.

La consultation d'un traité contemporain de Proust relatif à l' « organisation matérielle » du « travail intellectuel ${ }^{43}$ » laisse mesurer tout ce qui sépare l'écrivain d'une activité rationnelle qui faciliterait l'archivage et la remémoration de ses écrits. La souplesse, le caractère spontané et intuitif des procédés proustiens ouvrent sans doute la voie à une forme de mémoire involontaire. Ils permettent en tout cas une projection vers l'avenir de l'œuvre et une grande plasticité dans la transformation

38. Cicéron, que citent les éditeurs du cahier, distingue deux genres de beauté, la venustas, féminine et gracieuse, et la dignitas, majestueuse et masculine (De officiis, I, 130, cité ibid.). Dans La Défense et illustration de la langue française, Du Bellay emploie ce mot pour caractériser la langue grecque et lui comparer la langue française. Au XVII ${ }^{\mathrm{e}}$ siècle, son appartenance à la langue française fait débat et Gilles Ménage, pour le défendre, lui consacre deux chapitres de ses Observations sur la langue française. La base Frantext permet de constater que Chateaubriand l'emploie à propos de sa sœur Lucile d'une part, à propos de Venise d'autre part : dans les deux cas, il s'agit d'une forme de beauté morbide et sensuelle. Pétrus Borel l'emploie dans un contexte érotique (« Dina, la belle Juive », 1833). Le T.L.F., quant à lui, indique le sens premier de «Beauté comparable à celle de Vénus, pleine d'attraits sensuels, de séduction ineffable », en précisant bien « à propos d'une femme » (déjà dans le Littré). Hormis des sens dérivés, ce dictionnaire signale un second sens, « rare » : « éroticité, sensualité, volupté », en s'appuyant sur un exemple emprunté à Anatole France (L'Île des pingouins, 1908).

39. Marcel Proust, lettre à Reynaldo Hahn, Corr., t. VII, p. 42-43.

40. Toujours d'après la base Frantext. Après Proust, des occurrences chez Michel Tournier, Roland Barthes et Gabriel Matzneff peuvent suggérer une connotation homosexuelle de « vénusté », en dépit de la restriction de Cicéron, de Littré et du T.L.F.

41. Marcel Proust, lettres à Georges de Lauris, Corr., t. VIII, p. 324, 326 ; lettre à Abel Bonnard, Corr., t. XXI, p. 279.

42. Rhétorique à Hérennius, III, XXII, cité par Frances Yates, op. cit., p. 22. L'usage d'images érotiques est du reste avéré dans l'art de la mémoire (Lina Bolzoni, « Les phantasmes de l'Éros et les images de la mémoire », La Chambre de la mémoire, op. cit., p. 225-233).

43. Guyot-Daubès, L'Art de classer les notes et de garder le fruit de ses lectures et de ses travaux. Comment on organise son bureau, sa bibliothèque, Paris, P. Guyot éditeur, coll. "Bibliothèque d'éducation attrayante », 1891, p. 5. 
des fragments discursifs remémorés. La période ciblée, 1913-1914, correspond à la souffrance amoureuse causée par la fuite et la mort d'Alfred Agostinelli, qui retarde Proust dans son travail de préparation des tomes suivants, mais qui lui inspire le personnage d'Albertine et les développements narratifs ébauchés dans les Cahiers 71 et 54. Un grand apport de la psychologie expérimentale du $\mathrm{XX}^{\mathrm{e}}$ siècle est d'avoir montré que les phénomènes d'oubli ne s'expliquent pas seulement par l'écoulement du temps, par la durée qui effacerait progressivement les souvenirs, mais aussi et surtout par la superposition entre différentes choses à mémoriser, qui interfèrent les unes avec les autres. Or, Proust se trouve en 1913-1914 dans une situation d'interférence extrême. Il y a à cette époque, et pendant toutes les années qui suivront, à la fois un accroissement de la matière romanesque et une superposition des tâches cognitives de l'écrivain. Cela expliquerait pourquoi Proust commence alors à intensifier et à multiplier les procédés susceptibles de renforcer sa mémoire. 
Guillaume Perrier, docteur de l'université Paris Diderot-Paris VII, est l'auteur de La Mémoire du lecteur. Essais sur Albertine disparue et Le Temps retrouvé, Classiques Garnier, coll. «Bibliothèque proustienne », 2011. Il a coédité avec Raymonde Coudert, Textuel, $\mathrm{n}^{\circ}$ 45, « Proust. Surprises de la Recherche », 2004. Ses travaux sur Proust ont donné lieu à des publications dans les revues Écrire l'histoire, Revue internationale de philosophie, Études littéraires, ainsi que dans le Bulletin d'Informations proustiennes où il anime la rubrique « Notes de lecture ». Il a organisé en avril 2012 à l'ITEM une journée d'étude intitulée «La mémoire volontaire de l'écrivain ».

guillaume.perrier@gmail.com

\section{Résumés \\ Écriture et mnémotechnie : l'exemple des Cahiers Dux et Vénusté}

En même temps qu'il publie Du côté de chez Swann et qu'il travaille à la publication des tomes suivants, Proust rédige les premières versions de «l'épisode d'Albertine ». Ces manuscrits de 1913-1914 présentent, en marge du texte, un certain nombre de signes que l'écrivain adresse à sa propre mémoire pour ne rien perdre de ce qui surgit au cours de cette période d'intense vie créative mais aussi affective. Deux procédés sont étudiés ici : les amorces de notes du type « capitalissime » d'une part, les noms de pages et de cahiers comme « Dux » et « Vénusté » d'autre part. Ils sont situés dans la longue histoire des rapports entre écriture et mnémotechnie et plus particulièrement dans la tradition de « l'art de la mémoire ». Ils permettent d'envisager comment l'écrivain a développé les propriétés naturelles de sa mémoire pour créer une œuvre hors norme. Proust emploie ces procédés avec régularité mais aussi avec souplesse, de manière à capter le pouvoir des affects, de l'oubli et des réminiscences.

While Du côté de chez Swann was in the process of being published, and while Proust was working on the publication of the next volumes, he also wrote the first drafts of the "Albertine episode". Their 1913 and 1914 manuscripts contain alongside the text a number of signs, which were intended to aid the writer's own memory, so that he could retain everything that emerged during this period of intense feeling and creativity. This paper analyses two stylistic devices: the first part of notes like "capitalissime" on the one hand, titles of pages and notebooks like "Dux" and "Vénusté" on the other. They are situated in the long history of writing and mnemonics, and especially in the tradition of "the art of memory". They allow us to understand how the writer increased the natural properties of his memory in order to create a literary masterpiece. Proust uses these devices consistently, but also in a more flexible way, to capture the power of affect, forgetting and reminiscence.

Während der Publikation von Du côté de chez Swann und seiner Arbeit an den nachfolgenden Bänden hat Proust auch die ersten Entwürfe von „Albertine-Episode“ verfasst. Diese Handschriften von 1913-1914 enthalten am Rand des Textes eine Reihe von Zeichen, welche dem Autor zur Erinnerung dienten, um nichts von dem, was im Verlauf dieser Periode intensiver Kreativität und Emotionalität entstand, zu verlieren. Dieser Artikel untersucht zwei stilistische Methoden: einerseits den ersten Teil der von Proust als „,capitalissime" bezeichneten Notizen und andererseits die Titel der Seiten und Hefte wie beispielsweise „Dux" und „Vénusté“. Sie sind Teil der langen Geschichte der Verbindung zwischen Schreibprozess und Mnemotechnik, genauer der Tradition der ars memoriae und ermöglichen das Verständnis der Entwicklung des geistigen Eigentums des Schriftstellers im Prozess der Schaffung eines literarischen Meisterwerks. Proust verwendet diese Methoden auf eine stetige aber auch flexible Weise und fängt so die Kraft der Emotion, des Vergessens und der Erinnerung ein.
Al mismo tiempo que publica Por el camino de Swann y que trabaja en la publicación de los tomos siguientes, Proust redacta las primeras versiones del "episodio Albertine". Estos manuscritos de 1913-1914 contienen, al margen de los textos, una cierta cantidad de signos, que el escritor destina a su propia memoria para no perder nada de lo que va surgiendo a lo largo de ese periodo de intensa vida creativa, pero también afectiva. Se estudian aquí dos procedimientos: el inicio de notas del tipo "capitalísimo", por una parte, y por la otra, los nombres de páginas y de cuadernos, como "Dux" y "Vénusté". Estos procedimientos se integran en la larga historia de las relaciones entre escritura y mnemotécnica y más en particular, en la tradición del "arte de la memoria", y permiten estudiar la manera en que el escritor desarrolló las propiedades naturales de su memoria para crear una obra atípica. Proust utiliza estos procedimientos con la regularidad y la flexibilidad necesarias para captar el poder de los afectos, el olvido y las reminiscencias.

Negli stessi tempi in cui stampava Du côté de chez Swann, e lavorava alla pubblicazione dei tomi successivi, Proust tracciava le prime versioni de "l'épisode d'Albertine". I manoscritti, risalenti agli anni 1913-1914, conservano, ai margini del testo, una serie di richiami funzionali a favorire la memoria di quanto, in quel periodo di intensa vita creativa ed emotiva, gli andava accadendo. Il saggio, in questa prospettiva, analizza i due procedimenti stilistici che quelle pagine proustiane più peculiarmente documentano: la prima sezione di annotazioni del tipo "capitalissime", da un lato; i titoli di pagine e di fascicoli come "Dux"e "Vénusté" dall'altro. Non solo si situano nella lunga storia del rapporto tra scrittura e memoria, e più specificamente, nella tradizione dell'“arte della memoria"; ma permettono, anzi, di osservare in quali modi lo scrittore ha sviluppato le proprietà naturali della sua "memoria" per creare un'opera che andasse al di là della norma. Proust utilizza questi procedimenti con regolarità, ma anche con una qual certa duttilità, sì da catturare l'intensità delle emozioni, degli oblii e dei ricordi.

Enquanto publicava Du côté de chez Swann e trabalhava na publicação dos volumes seguintes, Proust escreveu as primeiras versões do "episódio de Albertine". Esses manuscritos de 1913-1914 apresentam, na margem do texto, uma série de signos inscritos pelo escritor com fins mnemónicos para nada perder do que lhe ocorre nesse período de intenso vida criativa, e também emocional. Dois processos são estudados aqui: esboços de notas como "capitalissime" e, por outro lado, nomes de páginas e de livros como "Dux" e "Memo". Situam-se eles na longa história da relação entre escrita e mnemotécnica, mais concretamente na tradição da "arte da memória". Permitem observar como o escritor desenvolveu as propriedades naturais da sua memória para criar uma obra de excepção. Proust usa estes métodos com regularidade mas também com flexibilidade, de modo a captar o poder das emoções, do olvido e das reminiscências. 